Article

\title{
Effect of Gap Flow on the Characteristics of Flow-Around and Flow-Induced Vibration for Two Circular Cylinders with Roughness Strips
}

\author{
Zuo-Mei Yang ${ }^{1,2}$, Lin Ding 1,2,*D, Qian-Yun Ye ${ }^{1,3}$, Lin Yang ${ }^{1,2}$ and Li Zhang ${ }^{1,2, *}$ \\ 1 Key Laboratory of Low-grade Energy Utilization Technologies and Systems of Ministry of Education, \\ Chongqing University, Chongqing 400044, China \\ 2 School of Energy and Power Engineering, Chongqing University, Chongqing 400044, China \\ 3 China southwest architectural design and research institute Corp. Ltd., Chengdu 610041, China \\ * Correspondence: linding@cqu.edu.cn (L.D.); lizhang@cqu.edu.cn (L.Z.); Tel.: +86-23-6510-3101 (L.D.)
}

Received: 28 July 2019; Accepted: 23 August 2019; Published: 2 September 2019

\begin{abstract}
In order to understand the gap flow between two cylinders, the characteristics of flow around two stationary cylinders and the flow-induced vibration of two staggered cylinders with roughness strips are numerically studied. The lift-drag responses, Strouhal number $(S t)$ and wake structure of two stationary cylinders in tandem, as well as the vibration response and vortex pattern of two oscillating staggered cylinders are analyzed. The results indicate that the spacing $d_{c}$ of two stationary cylinders at which the gap flow can be observed is different for different $R e$, and $d_{c}$ is $3 D$ when $R e=2000$ and $d_{c}=2.5 D$ at $R e=6000 \sim 14,000$. When the distance $d=d_{c}$, the force coefficient and $S t$ of two cylinders increase sharply. For the two oscillating staggered cylinders, there is a critical reduced velocity $U_{c}{ }^{*}=7$, which makes the amplitude magnitude relationship of the two cylinders change. With the change of the reduced velocity, the vibration frequencies of the two cylinders are consistent. When the staggered distance increases, the frequency difference of the two cylinders decreases. At the same inflow velocity, with the increase of staggered distance, a gap flow is formed between the two cylinders. When $T>0.6 \mathrm{D}$ and $U^{*}<8$, the gap flow becomes the main factor affecting the vibration of the two cylinders, which can be divided into the dominant region of gap flow.
\end{abstract}

Keywords: two cylinders; gap flow; stagger arrangement; flow-induced vibration; roughness strips

\section{Introduction}

In engineering practice, the bluff body is a common non streamlined structure. When the fluid flow around the bluff body, alternating vortex shedding occurs on both sides of the back surface of the bluff body, which causes the bluff body to be subjected to periodic hydrodynamic forces. When the bluff body is elastically supported, the periodic fluid force will excite the vibration of the bluff body, and the vibration of the bluff body will change the vortex shedding mode of the bluff body [1]. This phenomenon of structural vibration caused by the interaction between fluid and structure is called flow-induced vibration (FIV) [2,3]. A large number of practical engineering structures are bluff bodies, such as chimney towers, high-rise buildings, cooling towers, suspension bridges, offshore oil platforms, overhead cable lines, and submarine pipelines. FIV can cause fatigue damage to these buildings. Especially when the frequency of vortex shedding closes to the natural frequency of the bluff body, the vortex frequency will be locked-in. When lock-in occurs, the structure will be more seriously damaged by FIV, such as the collapse of Felibridge cooling tower caused by FIV. In many engineering and mechanical applications related to fluids, FIV is inevitably a major safety concern [4]. Therefore, research on the FIV of bluff bodies has great significance in theoretical research and practical applications. 
The FIV of a bluff body is a very complicated fluid-solid coupling process. Many studies have been done in this field, mainly focusing on the FIV characteristics and vibration control of the bluff body. The representative reviews of FIV of cylinders are by Williamson [5] and Bearman [6]. The control of FIV is embodied in two aspects. On the one hand, it is the suppression of vibration. Canpolat [7] found that rectangular grooves on a cylinder can effectively control flow through PIV experiments. Feng [8] used a water tunnel experimentation study and found that a synthetic jet can significantly change the scale of eddy current and wake mode. Lam [9] discussed the flow around a corrugated cylinder by large eddy simulation and experiment, and they controlled the vibration of the tube bundle in a heat exchanger by adding a corrugated cylinder to the tube bundle array. Zhu [10] and Song [11] studied the inhibition effect of small control rods on vortex-induced vibration (VIV). The former compared the VIV characteristics under different rod numbers, diameter ratios and gap ratios. It was found that when the attachment had nine control rods, the diameter ratio was 0.15 , and the clearance ratio was 0.6 , the inhibition effect of VIV was the best. The latter simulated the VIV characteristics of a cylinder with three small control rods at different angles of attack and clearance ratios. It was found that the inhibition effect was the best when the angle of attack was 45 and the clearance ratio was 0.9. Wu et al. [12] found a new method to control VIV: A twisted cylinder, which was applied to a semi-submersible offshore platform. Compared with a square cylinder, the amplitude of twisted cylinder decreased by $90 \%$ at the lock-in condition. On the other hand, Zhu et al. [13] studied the position and shape of the rough strip to control the VIV of the cylinder. It was found that the vibration could be effectively enhanced when the rough strip was located at $20^{\circ}$, and it could be restrained when it was located at $120^{\circ}$. At a highly reduced velocity, the rectangular and trapezoidal strips could promote the vibration, while the triangular and arc strips had little influence on the vibration. Chang [14], Ding [15-19] and Shan [20] et al. adopted the control method of surface-attached straight strip roughness to study the energy conversion of the FIV of a cylinder. It was found that the vibration of the cylinder with roughness strips was greatly enhanced under the condition of flow reduced velocity, and the kinetic energy of the fluid was more converted into the mechanical energy of vibration. In addition, with the rapid development of aerospace engineering, ocean engineering and wind engineering, more and more scholars begin to pay attention to the flow around multi-cylinders and FIV. Okajima [21] studied the vibration response of tandem cylinders in the direction of flow through a wind tunnel, one of which was fixed. The influence of the spacing ratio on vibration was analysed, and the wake structure of the cylinder was observed by the smoke visualization method. Zhou [22] researched the interaction between wakes of several parallel cylinders in turbulent flow. It was found that the Prandtl number, heat flux and vortex structure were affected by wake interaction and clearance flow deflection. Harichandan [23] developed a new solver to calculate the flow around multiple cylinders. The results show that the flow was greatly influenced by the Reynolds number and the spacing ratio. With the increase of the Reynolds number, the vortex shedding speed was faster, and the frequency of parallel three cylinders was larger than that of three cylinders in tandem. Chen et al. [24] studied the response and galloping of three tandem cylinders by numerical simulation. It was found that when the spacing ratio was small, the large-amplitude vibration of the cylinders was mainly affected by the wake-cylinder. While at the larger spacing ratio, the vibration response of the upstream cylinder was similar to that of an isolated single cylinder and was no longer affected by the downstream cylinder. Ding et al. [25] studied the FIV and energy recovery of three tandem cylinders with rough strips, and they found that the maximum amplitude of the upstream cylinder could reach $2.8 D$. The total power increased with the increasing of the reduced velocity of the water, and the power peaked at roughly 85.26 W. For the case of multiple cylinders, the flow field condition and the vibration of the cylinder were more complicated than the vibration of the single cylinder. The two cylinders are one of the simplest multi-cylinder forms and have been the basis for multi-cylinder research. Zhou et al. [26] collated and analysed the results of previous studies on the flow around two cylinders, and they found that the forces acting on the cylinder and the wake structure were influenced by many factors, such as the distance between the cylinders, the Reynolds number, the arrangement of the cylinders, and the 
size of the cylinder. Mysa et al. [27] studied the VIV of the two tandem cylinders. It was found that the maximum amplitude of the downstream cylinder of the two tandem cylinders was significantly larger than that of the single cylinder, and the critical buckling deceleration was between 5.0 and 6.0 at different spacing ratios. Tamimi et al. [28] simulated the FIV characteristics of a circular cylinder and a square cylinder arranged in tandem. The results were contrary to those of two cylinders in series. The square cylinder installed downstream showed a VIV-type response at different spacing ratios without galloping. Pearcey et al. [29] simulated the vibration of two cylinders with different diameters. It was found that the vortex shedding modes were different under different arrangement modes, and only the vibration of staggered arrangement was periodic and stable. Griffith et al. [30] numerically simulated the FIV response of two staggered cylinders and classified the relationship between gap distance, reduced velocity and vibration response, which were divided into a low reduced velocity zone, a critical state zone, a wake dominant zone, a gap flow dominant zone, and so on. Borazjani et al. [31] studied the VIV of two tandem cylinders in the wake-dominant region. It was found that the vibration amplitude and locking region of two tandem cylinders were larger than those of the isolated single cylinder. At a low reduced velocity, the vibration amplitude of the front cylinder exceeded the vibration amplitude of the rear cylinder. When the gap flow mechanism was induced, the amplitude of the VIV was greater than that of the one degree of freedom VIV. Liu and Jaiman $[32,33]$ simulated the FIV response of two parallel cylinders and found that the near wake instability was closely related to the gap flow and VIV.

It can be seen from the above that there are many factors affecting the FIV of two cylinders, among which the spacing ratio is one of the important factors. The influence of gap flow on the flow around two cylinders and the response of FIV has been found by earlier studies, but the specific effect is not clear. Therefore, in view of the effect of gap flow on FIV of two cylinders, the flow around the two cylinders, and the FIV characteristics in turbulent flow are studied in detail by a numerical method in this work in order to understand the influence mechanism of gap flow on two cylinders and to provide references for marine engineering related design. The physical model of two staggered cylinders is discussed in Section 2. The mathematical model and numerical calculation can be seen in Section 3. In Section 4, the amplitude, frequency and wake vortex pattern of cylinders are mainly analysed. The effects of the gap ratio in the downstream direction and the cross-flow direction on the flow characteristics of two cylinders are discussed. Some key conclusions are drawn in Section 5.

\section{Physical Model}

In this paper, a numerical method was adopted for the two cylinders with roughness strips. The physical model of the elastically supported two staggered cylinders is shown in Figure 1. The vibration direction of each cylinder was limited to the $y$-direction, which was perpendicular to the direction of inflow ( $x$-direction) and the axis of the cylinder. The cylinder in the vibration system was a flexible bluff body with a diameter of $D$ and a length of $L$. The elastic coefficient of the vibration system was $K$, and the structural damping caused by friction is $C$. The upstream surface of the cylinder was symmetrically attached with two rough bands along the axis. The thickness and coverage of each rough band were $0.847 \mathrm{~mm}$ and 16-degree. The angles of the two rough bands were different-the upstream cylinder was $\alpha= \pm 20^{\circ}$, and the downstream cylinder was $\alpha= \pm 30^{\circ}$ [15]. The Reynolds number range considered in this paper was $R e=2000 \sim 14,000$, and the corresponding reduced velocity $\left(U *=U /\left(f_{n} \cdot D\right), f_{n}=\sqrt{K /\left(m+m_{a}\right)} / 2 \pi\right.$ was the natural frequency) range was $2 \leq U^{*} \leq 14$. When the two tandem cylinders were fixedly arranged; the spacing in flow direction was $d=2 D \sim 7 D$; when the two cylinders were staggered with elastic supports, the cross-flow direction spacing was $T=0 \sim 1.0 D$, and the flow direction spacing $d$ was fixed at $2 D$. In this paper, the density and kinematic viscosity of liquid water at $15{ }^{\circ} \mathrm{C}$ were considered. The system parameters of the upstream and downstream cylinders were identical, as shown in Table 1. 


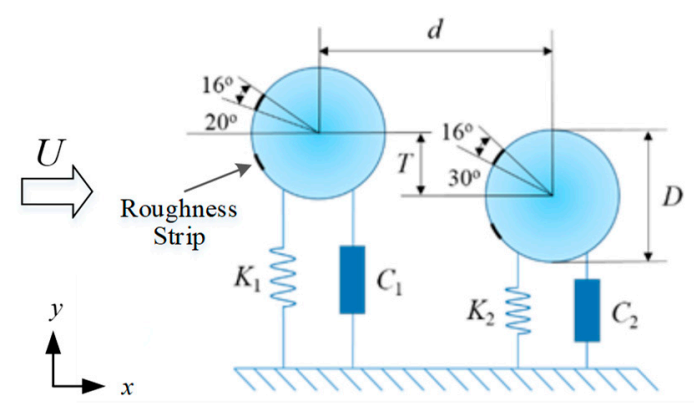

Figure 1. The physical model of two staggered circular cylinders with elastic support.

Table 1. Parameters of two cylinders with roughness strips oscillatory system.

\begin{tabular}{lc}
\hline \multicolumn{1}{c}{ Parameters } & Cylinder \\
\hline Structural damping per unit length: $C(\mathrm{~N} \cdot \mathrm{s} / \mathrm{m})$ & 1.36 \\
Spring stiffness per unit length: $K(\mathrm{~N} / \mathrm{m})$ & 80.38 \\
System quality per unit length: $m_{\text {osc }}(\mathrm{kg} / \mathrm{m})$ & 2.76 \\
Feature size: $D(\mathrm{~m})$ & 0.04 \\
Cylinder length: $L(\mathrm{~m})$ & 0.5 \\
Density of water at $15^{\circ} \mathrm{C}: \rho\left(\mathrm{kg} / \mathrm{m}^{3}\right)$ & 999.10 \\
Kinematic viscosity of water at $15^{\circ} \mathrm{C}: v\left(\mathrm{~m}^{2} / \mathrm{s}\right)$ & $1.14 \times 10^{-6}$ \\
\hline
\end{tabular}

\section{Mathematical Model and Numerical Calculation}

\subsection{Governing Equations}

The fluid flow was modelled by using two-dimensional unsteady Reynolds-averaged Navier-Stokes (RANS) equations together with the Spalart-Allmaras (S-A) turbulence model [34]. The incompressible fluid dynamics equations are:

$$
\begin{gathered}
\frac{\partial U_{i}}{\partial x_{i}}=0 \\
\rho \frac{\partial U_{i}}{\partial t}+\rho U_{j} \frac{\partial U_{i}}{\partial x_{j}}=-\frac{\partial P}{\partial x_{i}}+\frac{\partial}{\partial x_{j}}\left(2 \mu S_{j i}-\rho \overline{u_{j}^{\prime} u_{i}^{\prime}}\right)
\end{gathered}
$$

where $\rho$ and $P$ are the fluid density and flow field pressure, respectively, and the space and time coordinates are $x_{i}$ and $t$, respectively. $U_{i}$ is the mean flow velocity vector, and $S_{i j}$ is the mean strain-rate tensor.

$$
S_{i j}=\frac{1}{2}\left(\frac{\partial U_{i}}{\partial U_{j}}+\frac{\partial U_{j}}{\partial U_{i}}\right)
$$

Compared with the Navier-Stokes equation, there is a Reynolds stress tensor $\tau_{i j}=-\rho \overline{u_{i}{ }^{\prime} u_{j}^{\prime}}$ in the RANS equation, which is modelled through the Boussinesq eddy viscosity approximation as $2 \mu_{t} S_{i j}$, where $\mu_{t}$ is the turbulence eddy viscosity.

This paper adopts the S-A turbulent model with a good stability and small amount of computation, which is designed for wall-bound flow in aviation applications, has been shown to give acceptable results for a wide variety of situations, and is known for its stability. The S-A model is different from other one-equation models and algebraic models in the sense that it is a local model, which means the equation in one location does not depend on the solution at other points.

\subsection{Equation of Motion}

In this paper, the vibration system of two staggered cylinders can be simplified as a mass-springdamping (M-C-K) system with only one degree of freedom considered. The equation of motion of the oscillator is as follows: 


$$
m \ddot{y}+C \dot{y}+K y=F_{f l u i d, y}
$$

where $m$ is the total mass of the vibration system, consisting of the mass of the vibration cylinder and one third of the mass of the spring; $\ddot{y}, \dot{y}$, and $y$ represent the acceleration, velocity, and displacement of the oscillator perpendicular to the flow direction ( $y$ direction), respectively; $F_{f l u i d, y}$ is the fluid force acting on the surface of the cylinder in the y direction, which can be obtained by integrating the pressure on the surface of the cylinder and the viscous friction force; $C_{\text {system }} \dot{y}$ is the sum of all kinds of drags acting on the cylinder in the flow field; and $K y$ is the elastic force of spring acting on the cylinder. It should be noted that the linear viscous damping and linear stiffness was considered for the two cylinders FIV model.

The open source computational fluid dynamics (CFD) tool OpenFOAM-extend (Wikki Ltd., London, United Kingdom) was used to simulate the flow past two cylinders in the present study. A second-order Gauss integration scheme with a linear interpolation for the face-centered value of the unknown was used for the divergence, gradient, and Laplacian terms in the governing equations. The second-order backward Euler method was adopted for time integration. Thus, the numerical discretization scheme gave second order accuracy in space and time. A pressure implicit with the splitting of operators (PISO) algorithm was used for solving the momentum and continuity equations together in a segregated way. The coupling between flow equations and motion equation was strongly coupled. The equation of motion for the circular cylinder was solved using a second-order mixed implicit and explicit time integration scheme.

\subsection{Computational Domain and Grid Generation}

The calculation area was a two-dimensional rectangular area with a size of $(30 D+d) \times 16 D$, as shown in Figure 2. The entire domain included five boundaries: Inflow, outflow, top, bottom, and cylinders. The inflow velocity was considered to be uniform and constant. The distance from the cylinder to the outlet boundary was sufficiently long, and, thus, the zero gradient boundary condition had negligible influence on the near-wake region of the cylinder flow. According to the hypothesis, the outflow was fully developed, and the derivative of velocity along the flow direction was zero. Therefore, the exit boundary was the pressure outlet, the flow velocity normal gradient was zero, and the pressure was zero. The top and bottom were fixed wall boundaries. When the cylinder was fixed, and it was considered that the cylinder wall was a non-slip wall boundary condition. To match the dynamic mesh technique of topological change, a moving wall boundary condition was applied for the cylinder surface when the cylinder was elastically supported in this work.

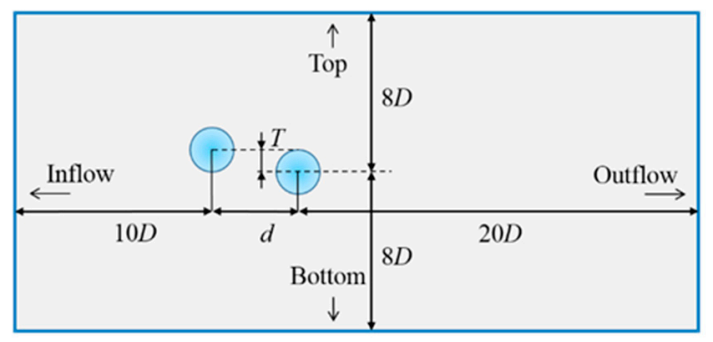

Figure 2. Computational domain $(30 D+d) \times 16 D$.

In order to describe the interaction force when the fluid flows around the cylinder with roughness strips for the cylinder with diameter $D$ and length $L$, the following formula was used to calculate the lift and drag coefficients:

$$
C_{d}=\frac{F_{\text {fluid }, x}}{\frac{1}{2} \rho U^{2} D L}
$$




$$
C_{l}=\frac{F_{f l u i d, y}}{\frac{1}{2} \rho U^{2} D L}
$$

The wake shedding frequency of a stationary flow around a circular cylinder is described by the dimensionless Strouhal number $(S t)$. The definition of $S t$ is as follows:

$$
S t=\frac{f_{v s} D}{U}
$$

where $f_{v s}$ is the frequency of vortex shedding.

Two-dimensional structured grids were used to calculate the static flow around two tandem cylinders. In order to capture the main characteristics of the flow field, it was necessary to encrypt the local mesh in the near-wall region with a large gradient of parameter variation. The verification of grid independence achieved good results, and, finally, the computation was carried out at medium density. The FIV of two staggered cylinders was calculated. In order to reduce the calculation error caused by grid distortion, the dynamic mesh technique of topological change was employed, and the calculation area near each cylinder with roughness strips was divided into $2 D \times 2 D$ square mesh sub-blocks. The cylinder was located in the centre of the sub-blocks, and the mesh density was increased. When FIV occurred, the mesh in the sub block moved along the cylinder wall without any mesh deformation. The GGI (Generalized Grid Interface) interface was used to connect the mesh sub-blocks with the upstream and downstream computational regions, and the topological mesh deformation technique was used to simulate the FIV of two cylinders. In addition, in order to converge within a reasonable time and obtain numerical solutions that meet the accuracy requirements, three different kinds of meshes (coarse, medium and fine) were selected to verify the grid independence. The calculation results in Table 2 show that the difference between the lift coefficient $\left(C_{l}\right)$ and the drag coefficient $\left(C_{d}\right)$ of the cylinder was very small with the change of the grid density, and the grid independence verification achieved good results. Therefore, the flow-induced vibration of two cylinders with roughness strips was simulated by using medium meshes. The computational area of the medium density grid is shown in Figure 3.

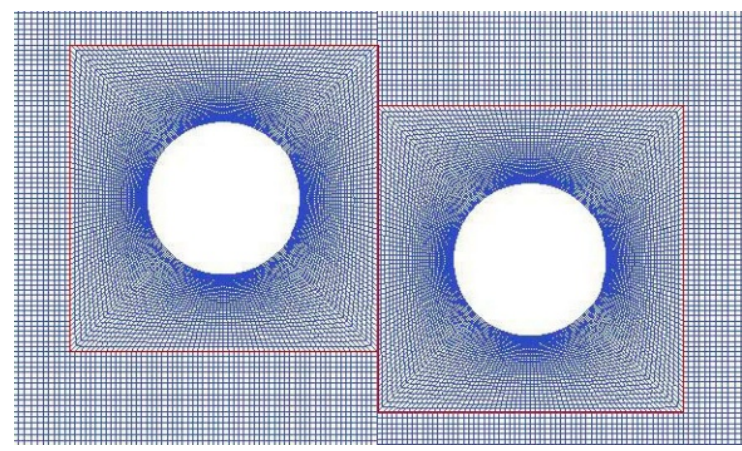

Figure 3. Medium grid: The close-up grid.

Table 2. Grid resolution study for two cylinders with roughness strips $\left(T / D=0, U^{*}=4\right)$.

\begin{tabular}{ccccc}
\hline \multirow{2}{*}{ Grid number $($ Axial $\times$ Radial) } & \multicolumn{2}{c}{ Drag coefficient $\left(C_{\boldsymbol{d}}\right)$} & \multicolumn{2}{c}{ Lift coefficient $\left(\boldsymbol{C}_{\boldsymbol{l}}\right)$} \\
\cline { 2 - 5 } & 1st & 2nd & 1st & 2nd \\
\hline Coarse $(180 \times 60)$ & 1.029 & -0.060 & 0.287 & 0.537 \\
Medium $(240 \times 70)$ & 1.039 & -0.065 & 0.299 & 0.561 \\
Fine $(360 \times 80)$ & 1.038 & -0.067 & 0.298 & 0.559 \\
\hline
\end{tabular}

\subsection{Model Validation}

In this paper, the FIV characteristics of the two cylinders in tandem were numerically studied and verified by the experimental data. The centre-to-centre distance of the cylinders was $2 D$. The results are shown in Figure 4. It can be seen from the figure that the amplitude change curves of the upstream 
and downstream cylinder all had three different branches (VIV initial branch, VIV upper branch, and galloping region), and the numerical simulation and experimental results [15] were basically consistent, which verifies the reliability and accuracy of the numerical simulation.

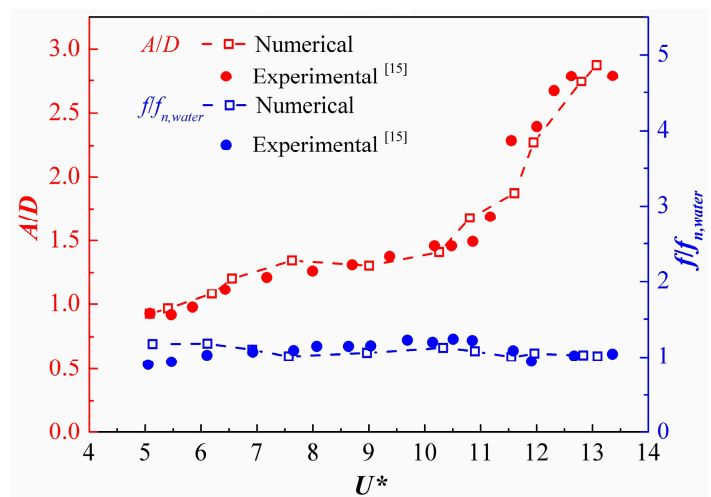

(a) Upstream cylinder

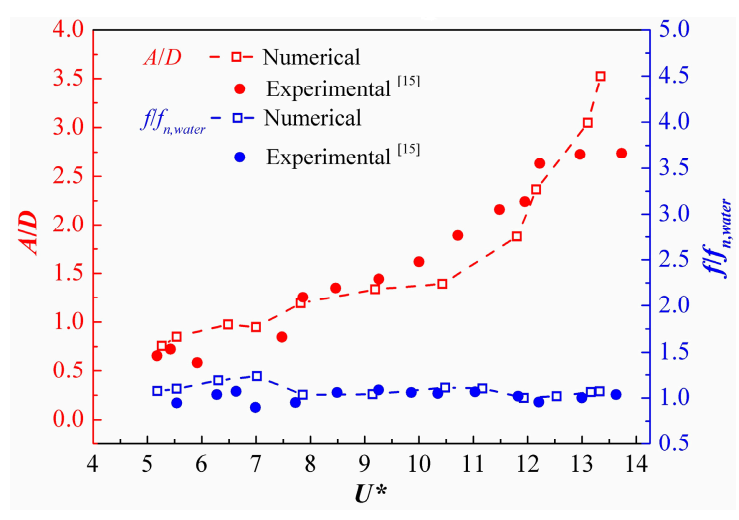

(b) Downstream cylinder

Figure 4. The amplitude and frequency responses of two tandem cylinders with roughness strips.

\section{Results and Discussion}

\subsection{Flow around Two Stationary Cylinders}

Considering that the spacing ratio along the flow direction affects the generation of the gap flow, the flow around two stationary cylinders with roughness strips in tandem with different spacing ratios under Reynolds number $R e=2000 \sim 14,000$ is numerically simulated in this section. The spacing ratio for gap flow is also discussed. Four representative Reynolds numbers $(R e=2000,6000,10,000,14,000)$ were selected for detailed analysis.

\subsubsection{Lift and Drag Coefficient}

Figure 5 shows the variation of the mean drag coefficient and the root mean square of the lift coefficient with the $x$-direction spacing ratio. According to Figure $5 \mathrm{a}$, the lifting force of the upstream and downstream cylinders was more consistent under different Reynolds numbers. In the case with $R e=2000$, the lift of the upstream and downstream cylinders suddenly increased to the maximum value when $d=3 D$ and then decreased slowly with the increase of the spacing ratio. The lift of the upstream cylinder was always smaller than that of the downstream cylinder. This is because the boundary layer separation of the upstream cylinder strengthened the vortex shedding strength of the downstream cylinder. When $R e=6000 \sim 14,000$, the lift force of the upstream and downstream cylinders was almost not affected by Reynolds number. The lift of upstream and downstream cylinders increased suddenly at $d=2.5 \mathrm{D}$, and then the lift of the upstream cylinders tended to stabilise with the increase of the spacing ratio, while the lift of the downstream cylinder first dropped sharply and then decreased slowly with the increase of the spacing ratio until the lift of the upstream cylinder was less than that of the upstream cylinder at $d>4.5 \mathrm{D}$. As the spacing ratio increased, the enhancement effect decreased continuously. From Figure 5b, the drag of the upstream cylinder was greater than that of the downstream cylinder and was always positive. For $R e=2000$, the drag of upstream cylinder increased suddenly when $d=3 D$ and then became stable with the increase of the spacing ratio. However, the drag of the downstream cylinder was negative when $d=2 D$ and suddenly increased to a positive value when $d=3 D$. Then, with the increase of the gap ratio, the drag increased slowly. As such, there was no obvious jump phenomenon. When $R e=6000 \sim 14,000$, the drag curves of upstream cylinder almost coincided, and all increased sharply when $d=2.5 \mathrm{D}$ and then became stable with the increase of the spacing ratio. The drag curve of the downstream cylinder appeared to have obvious jump phenomenon at $d=2.5 D$, and the drag value decreased first and then increased slowly with the increase 
of the spacing ratio. In conclusion, for the two stationary cylinders in tandem, there was a special spacing ratio, $d_{c}$, where the jump of force coefficient could be observed-when $R e=2000$, the $d_{c}=3 D$ and $R e \geq 6000, d_{c}=2.5 D$.

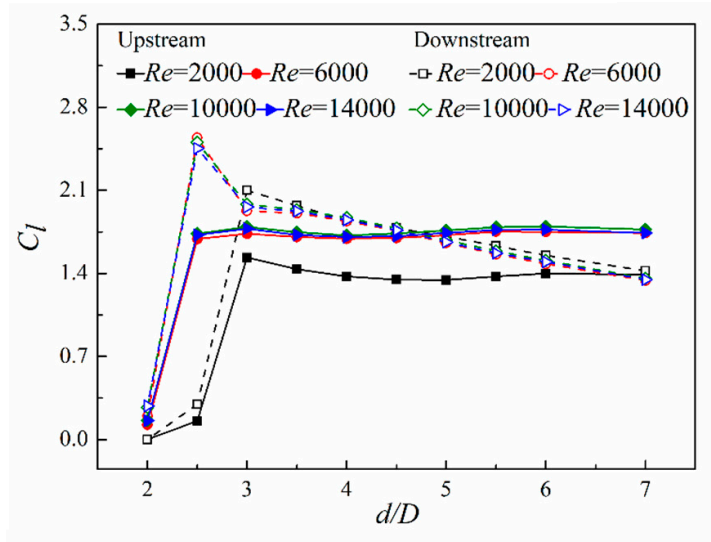

(a)

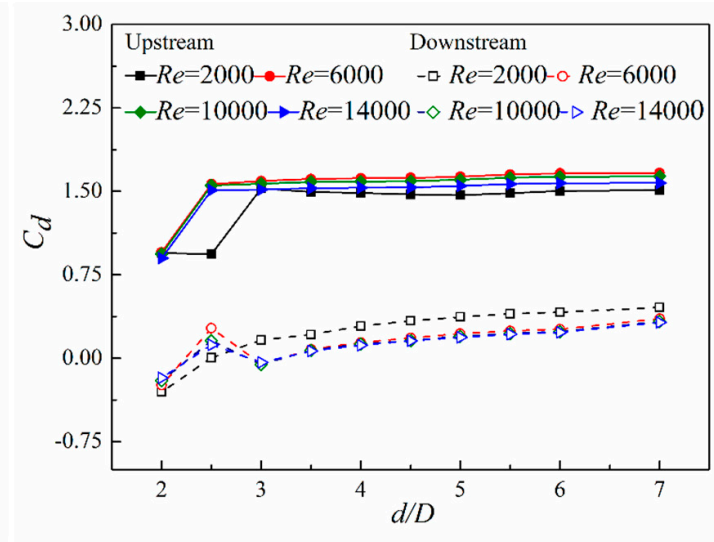

(b)

Figure 5. The lift and drag coefficients of two tandem cylinders with different spacing. (a) root mean square lift coefficient, (b) mean drag coefficient.

\subsubsection{Strouhal Number (St)}

In order to study the flow characteristics of two cylinders in detail, the $S t$ of the cylinders was analysed. From the results of the simulation, the vortex shedding frequencies of the two cylinders were equal, i.e., the number of $S t$ was equal for the flow around two fixed cylinders in tandem. Figure 6 shows the variation of the $S t$ with the spacing ratio at different Reynolds numbers. It can be seen that the frequency of vortex shedding increased with the increase of the spacing ratio. In the case of $d=2 D$ and $R e=2000$, it was hard to capture the vortex shedding between the two cylinders-that is, there was no vortex shedding frequency. When $d \leq 3 D$, St rose sharply from 0.15 to 0.21 with the increase of the spacing ratio; when $3 D<d \leq 4.5 D$, St increased slowly; when $d>4.5 D$, as the spacing ratio increased, the $S t$ gradually became stable and varied slightly around 0.225 . At $R e=2000$, the $S t$ suddenly increased at the spacing ratio $d_{c}=3 D$, and when $R e=6000 \sim 14,000$, the St suddenly changed at $d_{c}=2.5 D$, which was consistent with the lift and drag coefficients of the upstream and downstream cylinders found in Section 4.1.1. Overall, with the increase of gap distance, the $S t$ of the two cylinders gradually stabilized at 0.225 , which was slightly larger than that of the single cylinder (0.18-0.22) [35].

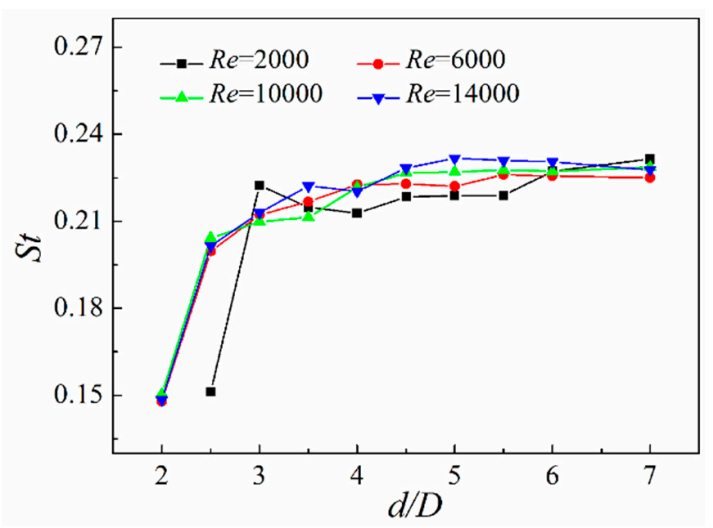

Figure 6. The Strouhal number (St) of two tandem circular cylinders with different spacing ratios.

\subsubsection{Wake Vortex Structure}

Figure 7 is a near-wake vortex pattern of the two tandem cylinders at $R e=2000$. As can be seen from Figure $7 \mathrm{a}$, in the case of $d=2 D$, the flow behind the cylinder presented a single slender structure, 
and the shear layer on the surface of the upstream cylinder completely enclosed the downstream cylinder. At this time, the two cylinders could be seen as a whole acting on the flow field, and no vortex shedding occurred in the two cylinders. When the spacing ratio increased to $d=2.5 D$, as shown in Figure $7 b$, the upstream cylinder did not form a vortex between the two cylinders. The shear layer was attached to the downstream cylinder surface, and the vortex shedding mode $2 S$ (S represents a single vortex) was formed at a distance behind the downstream cylinder. At $d=3 D$, as shown in Figure $7 \mathrm{c}$, the upstream cylinder began to shear, creating a vortex between the two cylinders and forming a gap flow. The vortex generated by the upstream cylinder passed through the side wall of the downstream cylinder, and the downstream cylinder wake formed a Karman vortex street. With the increase of the spacing ratio, steady vortices were gradually generated by the upstream cylinder between the two cylinders-that is, the gap flow. The gap flow between the two cylinders was cut off by the downstream cylinder when it moved downstream, which disturbed the flow pattern behind the downstream cylinder and presented the two-vortex shedding flow pattern shown in Figure 7d.

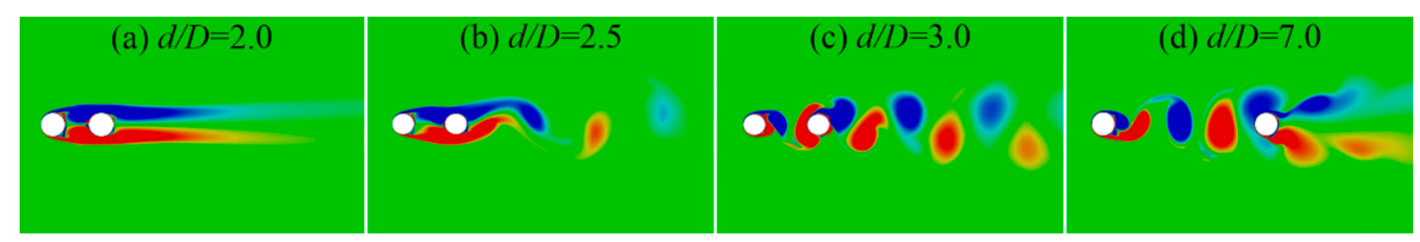

Figure 7. Vortex structure of the two stationary cylinders at different spacing ratios at $R e=2000$.

As shown in Figure 8, the near-wake vortex pattern of the two tandem cylinders at $R e=6000 \sim 14,000$ is presented. It can be seen from the diagram that the wake patterns of the two cylinders were similar when $R e=6000, R e=10,000$ and $R e=14,000$. The vortex pattern was $2 \mathrm{~S}$ for all the cases, which is the classical Kármán street, but the magnitude of vorticity had a slight difference. When $d=2.5 D$, the upstream cylinder could generate vortices between two cylinders, forming a gap flow. The generated vortices bypassed the front wall of the downstream cylinder without being destroyed and could be bypassed along the wall, producing the regular vortex street shown in Figure 8a. When the spacing ratio increased to $d=3.0 D$, as shown in Figure $8 \mathrm{~b}$, the wake mode behind the downstream cylinder changed from the single vortex shedding mode to the double vortex shedding mode, resulting in a sharp drop in the lift and drag of the downstream cylinder. With the increase of the spacing ratio, the downstream cylinder was greatly affected by the gap flow between the two cylinders, which can be seen from Figure 8c. When the spacing ratio continued to increase to $d=7.0 D$, the wake state of the upstream cylinder tended to be a single cylinder due to the large gap distance, as can be seen from Figure $8 \mathrm{~d}$. The energy of the vortex shedding from the upstream cylinder was small at downstream, which made the wake of the downstream cylinder unable to form an obvious vortex street and weakened the interaction between the two cylinders. The difference is that with the increase of Reynolds number, the vortex shedding frequency of the cylinder increased.

It can be seen from the above that the spacing ratio affected the flow characteristics of the two tandem cylinders. As the spacing ratio increased, a gap flow occurred between the two cylinders. Under different Reynolds numbers, the spacing ratio of the gap flow was different. When $R e=2000$, the spacing ratio of the gap flow was $d_{c} / D=3$; when $R e=6000 \sim 14,000$, the spacing ratio of the gap flow was $d_{c} / D=2.5$. At the spacing ratio $d_{c}$, the lift coefficient, drag coefficient, and St of the two cylinders had a sudden rise, which indicates that the generation of gap flow had a great influence on them.

\subsection{Flow Induced Vibration of Two Staggered Cylinders}

According to the analysis results of the flow around the two stationary cylinders with roughness strips in tandem, there was no gap flow between the two cylinders at different Reynolds numbers when the distance between the two cylinders was $d=2 D$. Therefore, in the FIV calculation region, the spacing of the two cylinders were set to $2 D$ along the flow direction. 


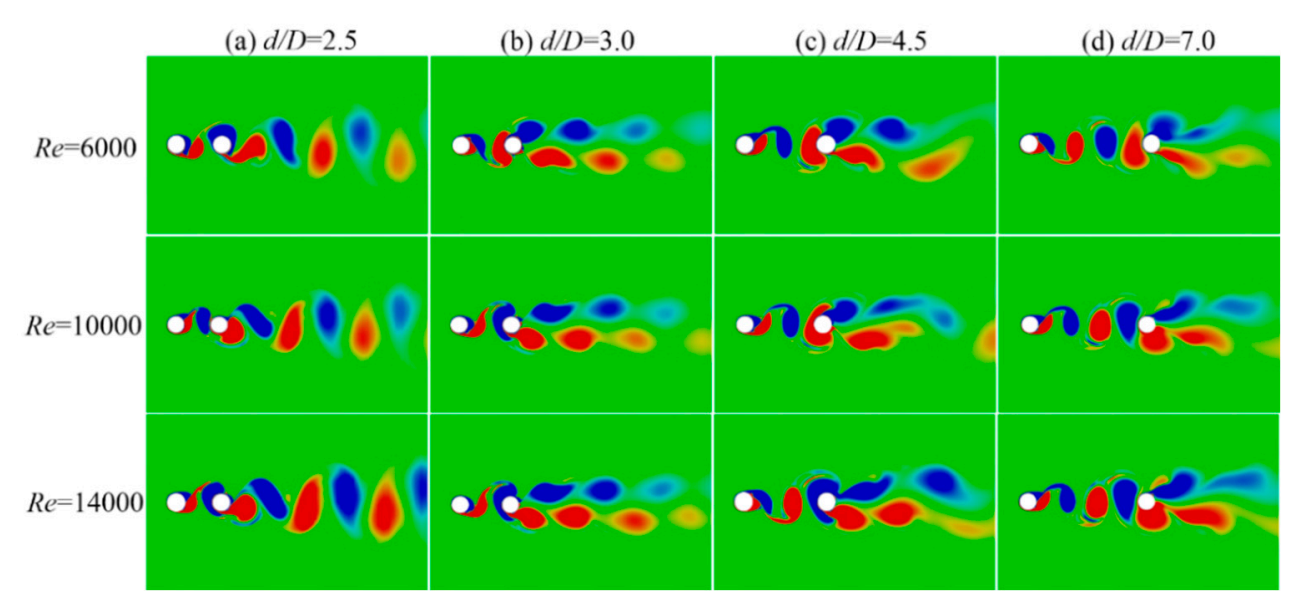

Figure 8. Vortex structure of the two stationary cylinders at different spacing ratios at $R e=6000 \sim 14,000$.

\subsubsection{Vibration Characteristics Partition}

The time-domain and frequency-domain characteristic curves of displacement of the two cylinders were analysed. It was found that the vibration characteristics of the cylinders changed with the gap distance and reduced velocity. The time-domain and frequency-domain curves are depicted in Figure 9. The amplitude curves were directly output through CFD calculation, and the frequency curves were obtained by the fast Fourier transform (FFT). When the reduced velocity was small $\left(U^{*}=3\right)$, the two cylinders were of periodic vibration mode. The amplitude-time curve of the cylinder presented a standard sinusoidal curve with very small amplitude. The two cylinders vibrated the same frequency, and the main frequency of the vibration was single and stable, as shown in Figure 9a. With the slight increase of staggered spacing and reduced velocity, the two cylinders were still in the mode of periodic vibration. The difference is that the mode of periodic vibration was period-doubling referred by Griffith [30], as shown in Figure 9b. When the staggered spacing increased to $T / D=0.8$, the downstream cylinder was still in periodic vibration mode, while the upstream cylinder was in multi-periodic vibration mode. Both vibrated together at the same frequency, as shown in Figure 9c. When the reduced velocity increased to a certain extent $\left(U^{*}=6\right)$, the vibration mode of the cylinder changed to quasi-periodicity mode. At this time, the vibration of the downstream cylinder was stronger and its displacement was larger, as shown in Figure 9d.
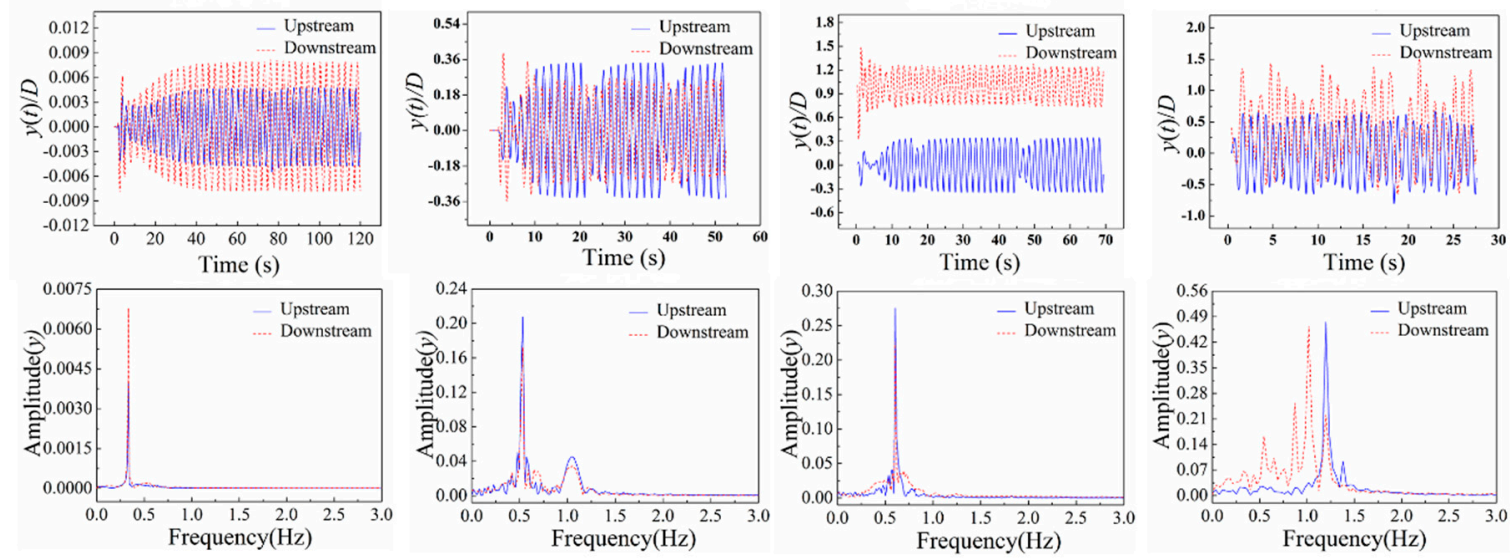

(a) $T / D=0, U^{*}=3$

(b) $T / D=0, U^{*}=3$

(c) $T / D=0.8, U^{*}=4$

(d) $T / D=0, U^{*}=9$

Figure 9. The time-domain and frequency-domain characteristic curves: (a) $T / D=0, U^{*}=3 ;(\mathbf{b}) T / D=0$, $U^{*}=4 ;(\mathbf{c}) T / D=0.8, U^{*}=4 ;$ and $(\mathbf{d}) T / D=0, U^{*}=9$. 


\subsubsection{Amplitude and Frequency Responses}

Amplitude and frequency are very important parameters to describe the motion characteristics and flow-induced vibration response of oscillators. The transition of the different FIV states of the cylinder is accompanied by changes in the response of different frequencies. The amplitude adopted in this paper was obtained by taking the root mean square value of the absolute value of all the displacements in the stable vibration phase of the cylinder. The root mean square of displacement reflected the intensity of vibration of a cylinder. The vibration frequency was obtained by FFT. The amplitude and frequency responses of the two cylinders under different spacing ratios are presented in Figures 10 and 11.

From Figure 10, it can be seen that the two cylinders underwent initial, upper and lower branches within the calculated reduced velocity. At a low reduced velocity $\left(U^{*} \leq 5\right)$, the amplitude of the upstream cylinder increased with the increase of the reduced velocity. At this time, the upstream cylinder vibration was located in the initial branch, in which the flow-induced vibration of the cylinder could not be clearly observed to be strengthened or suppressed. At this time, the velocity was low, and the gap distance had little effect on the upstream cylinder amplitude. When $5<U^{*}<11$, the FIV of the upstream cylinder was in the upper branch with high amplitude, and the maximum amplitude was obtained at $U^{*}=6$. The vibration of the upstream cylinder became chaotic, and the vibration was located in the lower branch when $U^{*}>11$. For the downstream cylinder, the initial branch, the upper branch and the lower branch were located at $U^{*} \leq 7,7<U^{*}<11$ and $U^{*}>11$, respectively, and the maximum amplitude occurred at $U^{*}=11$. Due to the influence of the upstream cylinder, the upper branch region of the downstream cylinder was narrower than the upper branch region of the upstream cylinder. When $U^{*}>11$, the vibration of the downstream cylinder became chaotic, and at this time, the vibration of the downstream cylinder tended to develop towards the lower branch. Besides, it was found that the upstream cylinder amplitude decreased slowly with the increase of the reduced velocity when the reduced velocity reached to 6 , while the downstream cylinder vibration amplitude increased. In the vicinity of $U^{*}=7$, the amplitude curves of the upstream and downstream cylinders intersected. In the case of $U^{*}<7$, the amplitude of the upstream cylinder was larger than that of the downstream cylinder. After $U^{*}>7$, the amplitude of the upstream cylinder was smaller than that of the downstream cylinder. This is because when the reduced velocity was large, the vibration between the two cylinders was stimulated, and the interaction between the two cylinders was increased. The downstream cylinder was affected by the upstream cylinder vortex shedding, and the vibration of the downstream cylinder was strengthened. Therefore, there was a critical reduced velocity. Before reaching the critical reduced velocity, the amplitude of the upstream cylinder was higher than that of the downstream cylinder. After the critical reduced velocity exceeded, the amplitude of the upstream cylinder was lower than that of the downstream cylinder.

As can be seen from Figure 11, the frequency ratio of the two cylinders was close, and the frequency ratio of the downstream cylinder was smaller than that of the upstream cylinder under the same working condition. With the increase of the spacing ratio, the difference of frequency ratios for the two cylinders decreased gradually. The vibration of the two cylinders basically coincided at $T / D=1$. The interaction between the two cylinders decreased at a large spacing ratio, and the two cylinders vibrated at almost the same frequency. The upstream cylinder frequency locking phenomenon appeared at $U^{*}=5 \sim 6$, and the amplitude corresponded to the maximum. When $T / D$ was smaller and larger, the downstream cylinder frequency locking occurred at $U^{*}=11 \sim 12$ but did not occur when $T / D=0.4 \sim 0.6$. 

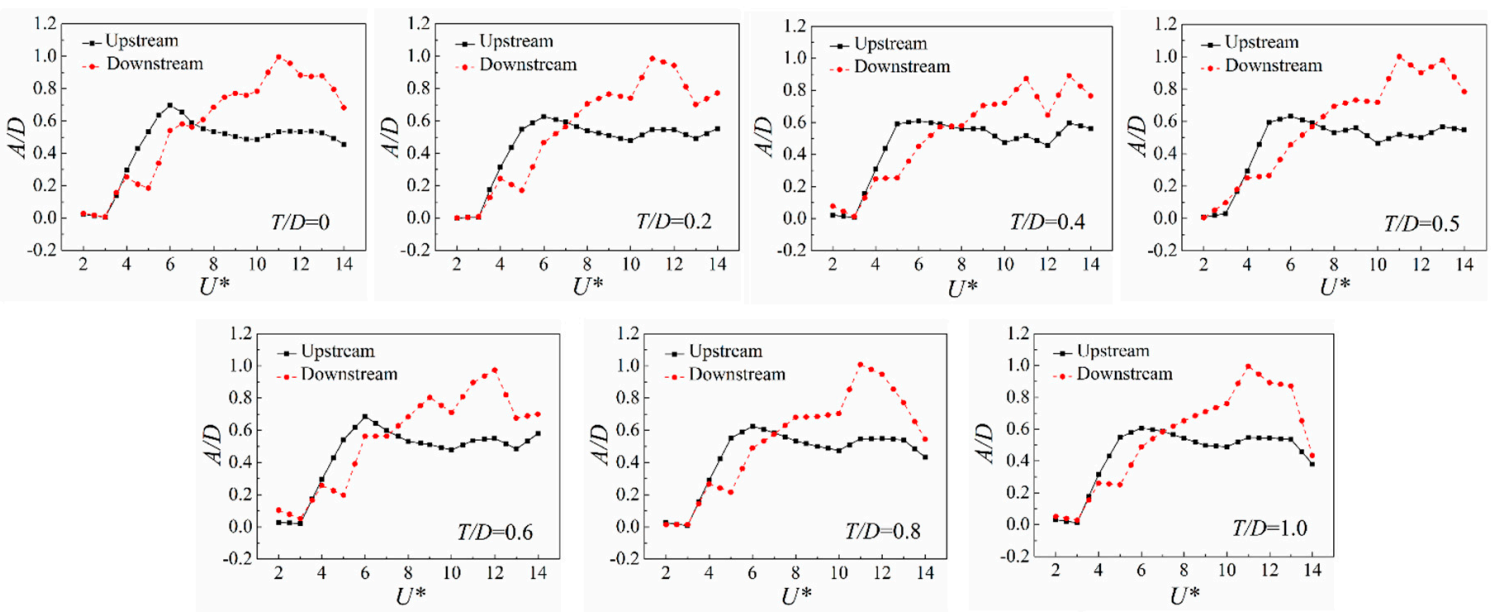

Figure 10. The amplitude responses of two cylinders under different spacing ratios.
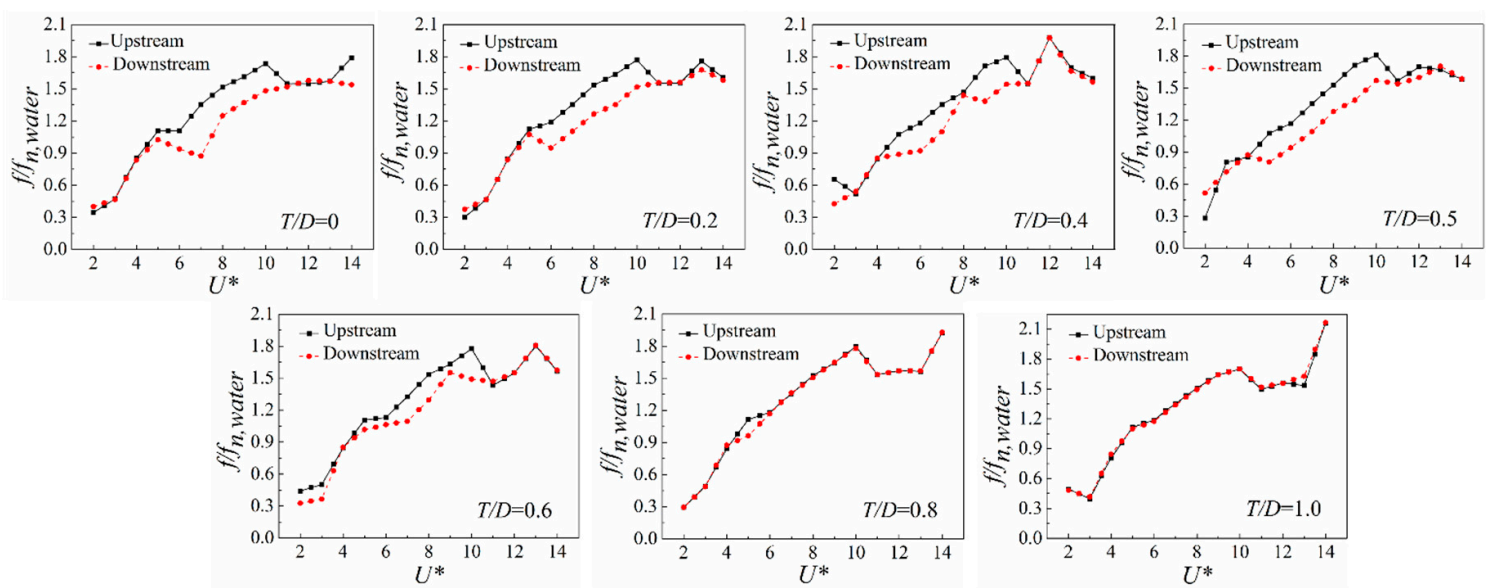

Figure 11. The frequency responses of two cylinders under different spacing ratios.

\subsubsection{Wake Vortex Structure}

The amplitude and frequency of the two cylinders can be determined by the fluid force on the two cylinders, which is closely related to the vortex shedding behind the bluff body. At the same time, the vortex generation and shedding process can be reversed by the vibration characteristics of two cylinders. The amplitude frequency response of FIV of two cylinders is closely related to its wake structure. When the vibration branch changes with the velocity of the incoming flow, the wake structure will also undergo modal transformation, among which are the typical wake vortex modes S, P (Pair), P+S (A pair of vortices add a single vortex), and so on. This section mainly selects four different working conditions to analyse the calculation results.

The wake vortex structure of two cylinders with roughness strips at different reduced velocities at $T / D=0$ is shown in Figure 12. As can be seen from Figure 12a, in the case with small reduced velocity $\left(U^{*}=2\right)$, the two cylinders hardly vibrated, and the upstream cylinders could not form vortices. The downstream mode of the vortices was S. With the increase of the reduced velocity, the upstream cylinder could form a vortex, but it could not fall off. The vortices which could not fall off from the upstream cylinder were then attached to the surface of the downstream cylinder, which affected the downstream cylinder and in turn promoted its own vibration. When the reduced velocity increased to $U^{*}=6$, the gap flow occurred between the two cylinders, which means that the interaction between the two cylinders was strengthened. After that, the amplitude of the downstream cylinder was larger than that of the upstream cylinder. As shown in Figure 12b, the vortex of shedding in P mode from the upstream cylinder was attached to the downstream cylinder surface again Meanwhile, enveloping 
the $\mathrm{S}$ mode vortex formed by the downstream cylinder and behind the downstream cylinder, the $\mathrm{P}+\mathrm{S}$ mode was shedding. When the reduced velocity continued to increase, the falling vortices from the rear of the upstream cylinder were still attached to the surface of the downstream cylinder and fell off with the downstream cylinder vortices. The falling mode was P and S, as shown in Figure 12c. When the reduced velocity increased to $U^{*}=11$, the vortices formed by the upstream cylinder were difficult to fall off. The unsealed vortices acted on the surface of the downstream cylinder to promote the vibration of the downstream cylinder. The backward vortices of the downstream cylinder were similar to the P mode, as shown in Figure $12 \mathrm{~d}$. After $U^{*}>11$, the shape and size of vortices were quite different. The downstream cylinder generally shed off in the P and S mode, as shown in Figure 12e.

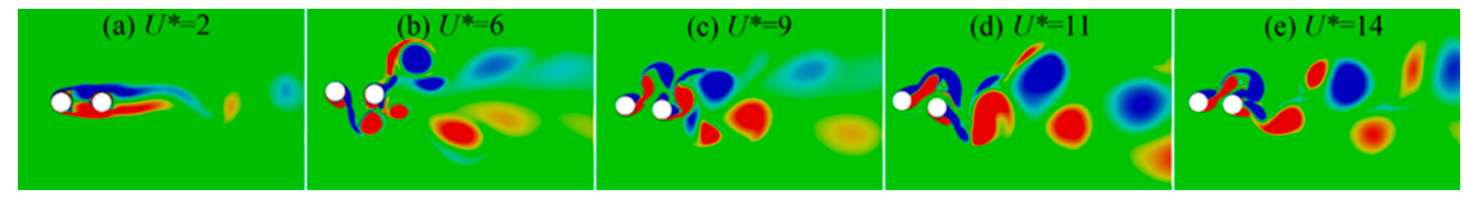

Figure 12. The wake vortex structure of two cylinders with roughness strips at different reduced velocities at $T=0$.

Figure 13 shows the wake vortex structure of the two cylinders with roughness strips at different reduced velocities at $T=0.4 D$. From Figure 13a, it can be seen that the upstream and downstream cylinders hardly vibrated at a low reduced velocity, but there was a tendency of gap flow between the two cylinders. As shown in Figure 13b, the upstream cylinder could form completely vortices in the P mode and was hardly affected by the downstream cylinder. The vortex formed independently by the downstream cylinder merged with the vortex shedding of the upstream cylinder, and it still shed in the P mode at the rear. At $U^{*}=9$, the upstream and downstream cylinders fell off in the P mode, but the two cylinders did not merge near the wake. The P mode is shown behind the downstream cylinder in Figure 13c. It is worth noting that, in the case of $U^{*}=11$, the downstream cylinder vortex shedding was more complicated than that under other conditions as shown in Figure 13d. The fluid-structure interaction between the downstream cylinder and fluid was enhanced, the FIV of the downstream cylinder was strengthened, and its amplitude also increased. As shown in Figure 13e, when the reduced velocity increased to 12 , the vortex shedding mode behind the downstream cylinder could be observed to change from $\mathrm{P}$ and $\mathrm{S}$ to $\mathrm{S}$, and the wake region behind the cylinder became narrow and slender. As a result, the pressure fluctuation in the wake region decreased, the FIV of the downstream cylinder was weakened, and its amplitude also decreased.
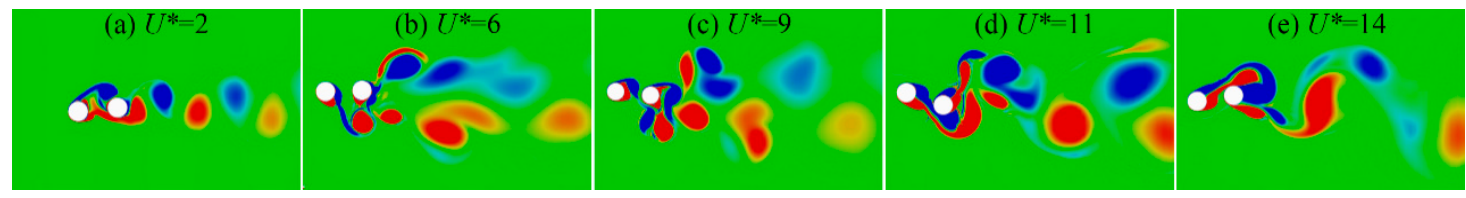

Figure 13. The wake vortex structure of two cylinders with roughness strips at different reduced velocities at $T=0.4 D$.

At $T=0.6 D$, the wake vortex structure of two cylinders with roughness strips at different reduced velocities is depicted in Figure 14. From Figure 14a-c, it can be clearly seen that the vortex shedding mode of the downstream cylinder changed from $S$ to $P$ and finally to $P$ and $S$, and the upstream cylinder shedding mode changed from $S$ to $P$. During this period, the number of vortex shedding increased and the pressure fluctuations in the wake area increased. Due to the influence of upstream vortex shedding, the fluid-solid interaction between the downstream cylinder and fluid was promoted, and the vibration of the downstream cylinder was strengthened. It can be observed from Figure 14d, e that the wake of the upstream cylinder became longer at a high reduced velocity. At this time, besides the inflow velocity, the downstream cylinder was the main factor affecting the upstream cylinder vibration. 
In the classical FIV of a single cylinder, when the reduced velocity is greater than 11, the vibration of the cylinder is in the lower part of the support, and the amplitude of the cylinder is very small; however, the existence of the downstream cylinder makes the boundary layer of the upstream cylinder separate ahead of time, and the upstream cylinder vibration maintains at a higher amplitude. The gap flow between two cylinders promotes the upstream cylinder vibration and suppresses the downstream cylinder vibration. The vortex shedding mode of the downstream cylinder could be approximately regarded as $\mathrm{S}$ at the far wake.
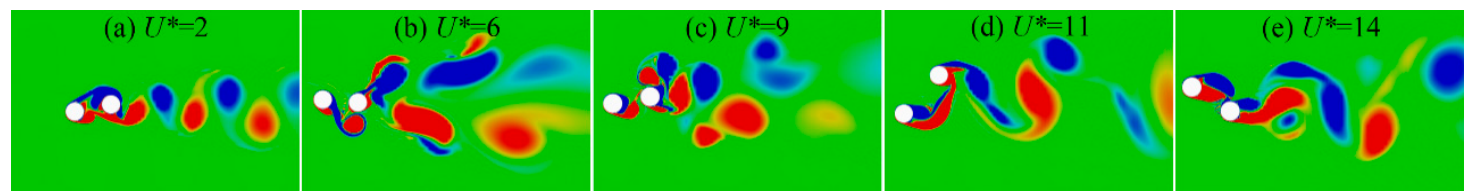

Figure 14. The wake vortex structure of two cylinders with roughness strips at different reduced velocities at $T=0.6 D$.

Figure 15 shows the wake vortex structure of two cylinders with roughness strips at different reduced velocities at $T=1.0 D$. This was similar to the vortex shedding mode at $T=0.6 D$. This is because the two cylinders were located in the dominant area of the gap flow when $U^{*}<8,1.0 D \geq T$ $\geq 0.6 \mathrm{D}$. When the distance was small, the staggered distance had little effect on the vibration of two cylinders. For $U^{*}=9$, the upstream cylinder shed in the P mode, and the downstream cylinder shed along with the upstream cylinder shedding in near wake, thus presenting a $\mathrm{P}$ and $\mathrm{S}$ mode, which lasted only for a short time and finally became the $S$ mode, as shown in Figure 15c. From Figure 15d, e, it can be seen that when the reduced velocity increased to a critical value, the gap flow between the upstream cylinder and the downstream cylinder changed from a complete vortex to a fine flow, resulting in a narrow near wake of the downstream cylinder, a smaller pressure fluctuation in the wake region, and a suppression of its vibration.
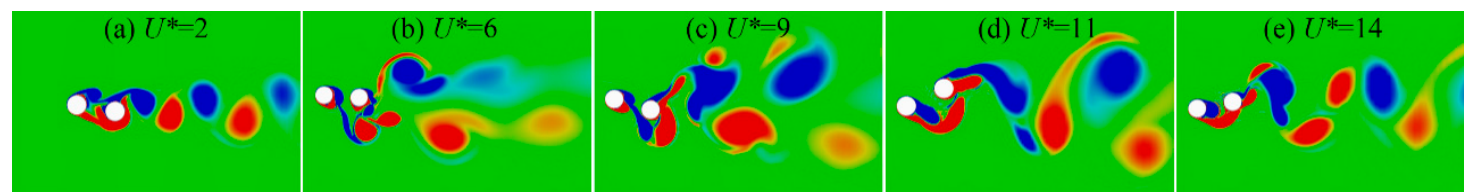

Figure 15. The wake vortex structure of two cylinders with roughness strips at different reduced velocities at $T=1.0 D$.

\section{Conclusion}

In this paper, the characteristics of flow around two cylinders with roughness strips and their FIV under turbulent condition were studied in detail by a numerical method. The amplitude, frequency, and wake vortex pattern of the cylinders were analysed. The influence of the gap distance ratio along flow direction and cross flow direction on FIV characteristics of two cylinders was discussed. The influence mechanism of gap flow on two cylinders was understood. The main conclusions are as follows:

(1) For the two stationary cylinders, the spacing $d c$ at which the gap flow can be observed is $3 D$ when $R e=2000, d_{c}=2.5 D$ at $R e=6000 \sim 14,000$. When gap distance ratio $d=d_{c}$, a gap flow is generated, and the lift-drag coefficient and the St of the cylinders increase sharply.

(2) For the two staggered cylinders, the vibration modes include: Periodic vibration mode (small reduced velocity), double-periodic vibration mode (moderate reduced velocity and small staggered distance), multi-periodic vibration mode (moderate reduced velocity and large staggered distance) and quasi-periodic vibration mode (large reduced velocity).

(3) When the staggered distance is changed, the amplitude of the upstream cylinder changes slightly, and the maximum amplitude can be obtained at $U^{*}=6$. The amplitude of the downstream 
cylinder varies significantly, and its maximum amplitude is obtained at $T=0.6 D$ and $U^{*}=12$. The variation of vibration frequency of two cylinders is consistent. With the increase of staggered distance, the vibration frequencies of the two cylinders get closer.

(4) At the same inflow velocity, the gap flow between the two cylinders is formed as the staggered distance increases. When $T>0.6 D$ and $U^{*}<8$, gap flow becomes the main factor affecting the vibration of the two cylinders, which can be divided into the dominant region of gap flow. In other regions, the two cylinders can be approximated as the arrangement in series.

Author Contributions: Conceptualization, Z.-M.Y., L.D., and Q.-Y.Y.; methodology, Z.-M.Y., L.D., Q.-Y.Y., and L.Y.; validation, Z.-M.Y. and Q.-Y.Y.; formal analysis, Z.-M.Y. and L.D.; investigation, Z.-M.Y., Q.-Y.Y., and L.Y.; resources, L.D. and L.Z.; data curation, Z.-M.Y. and L.D.; writing-original draft preparation, Z.-M.Y. and L.D.; writing—review and editing, L.Z. and Q.-Y.Y.; supervision, L.Z.; project administration, L.D.; funding acquisition, L.D.

Funding: This research was funded by National Natural Science Foundation of China, grant number 51776021.

Acknowledgments: The authors acknowledge the help from VIVACE group of Marine Renewable Energy Laboratory of the University of Michigan, and special thanks to Michael M. Bernitsas and Eun Soo Kim.

Conflicts of Interest: The authors declare no conflict of interest.

\section{References}

1. Xu, W.H.; Qin, W.Q.; Gao, X.F. Experimental Study on Streamwise Vortex-Induced Vibration of a Flexible, Slender Cylinder. Appl. Sci. Basel 2018, 8, 311. [CrossRef]

2. Prasanth, T.K.; Mittal, S. Vortex-induced vibrations of a circular cylinder at low Reynolds numbers. J. Fluid Mech. 2008, 594, 463-491. [CrossRef]

3. Chin, W.K.; Ong, Z.C.; Kong, K.K.; Khoo, S.; Huang, Y.H.; Chong, W. Enhancement of Energy Harvesting Performance by a Coupled Bluff Splitter Body and PVEH Plate through Vortex Induced Vibration near Resonance. Appl. Sci. Basel 2017, 7,921. [CrossRef]

4. Nguyen, T.; Koide, M.; Yamada, S.; Takahashi, T.; Shirakashi, M. Influence of mass and damping ratios on VIVs of a cylinder with a downstream counterpart in cruciform arrangement. J. Fluids Struct. 2012, 28, 40-55. [CrossRef]

5. Williamson, C.H.K.; Govardhan, R. Vortex-induced vibrations. Annu. Rev. Fluid Mech. 2004, 36, 413-455. [CrossRef]

6. Bearman, P.W. Circular cylinder wakes and vortex-induced vibrations. J. Fluids Struct. 2011, 27, 648-658. [CrossRef]

7. Canpolat, C.; Sahin, B. Influence of single rectangular groove on the flow past a circular cylinder. Int. J. Heat Fluid Flow 2017, 64, 79-88. [CrossRef]

8. Feng, L.H.; Ran, L.K. Wavelet multi-scale analysis of the circular cylinder wake under synthetic jets control. Int. J. Heat Fluid Flow 2018, 69, 73-82. [CrossRef]

9. Lam, K.; Lin, Y.F.; Zou, L.; Liu, Y. Experimental study and large eddy simulation of turbulent flow around tube bundles composed of wavy and circular cylinders. Int. J. Heat Fluid Flow 2010, 31, 32-44. [CrossRef]

10. Zhu, H.; Yao, J. Numerical evaluation of passive control of VIV by small control rods. Appl. Ocean Res. 2015, 51, 93-116. [CrossRef]

11. Song, Z.; Duan, M.; Gu, J. Numerical investigation on the suppression of VIV for a circular cylinder by three small control rods. Appl. Ocean Res. 2017, 64, 169-183. [CrossRef]

12. Wu, C.H.; Jaiman, R.K.; Lim, T.B.A.; Kang, C.W.; Ma, S. A new passive control technique for the suppression of vortex-induced motion in deep-draft semisubmersibles. Appl. Ocean Res. 2018, 80, 79-100. [CrossRef]

13. Zhu, H.; Gao, Y.; Zhou, T. Flow-induced vibration of a locally rough cylinder with two symmetrical strips attached on its surface: Effect of the location and shape of strips. Appl. Ocean Res. 2018, 72, 122-140. [CrossRef]

14. Chang, C.C.; Kumar, R.A.; Bernitsas, M.M. VIV and galloping of single circular cylinder with surface roughness at $3.0 \times 104 \leq 1.2 \times 105$. Ocean Eng. 2011, 38, 1713-1732. [CrossRef]

15. Ding, L.; Bernitsas, M.M.; Kim, E.S. 2-D URANS vs. experiments of flow induced motions of two circular cylinders in tandem with passive turbulence control for 30,000 $<\operatorname{Re}<105,000$. Ocean Eng. 2013, 72, 429-440. 
16. Ding, L.; Zhang, L.; Wu, C. Numerical Study on the Effect of Tandem Spacing on Flow-Induced Motions of Two Cylinders with Passive Turbulence Control. J. Offshore Mech. Arct. Eng. 2017, 139, 021801. [CrossRef]

17. Ding, L.; Zhang, L.; Wu, C.; Mao, X.; Jiang, D. Flow induced motion and energy harvesting of bluff bodies with different cross sections. Energy Convers. Manag. 2015, 91, 416-426. [CrossRef]

18. Ding, L.; Zhang, L.; Kim, E.S. URANS vs. experiments of flow induced motions of multiple circular cylinders with passive turbulence control. J. Fluids Struct. 2015, 54, 612-628. [CrossRef]

19. Ding, L.; Zhang, L.; Bernitsas, M.M.; Chang, C.C. Numerical simulation and experimental validation for energy harvesting of single-cylinder VIVACE converter with passive turbulence control. Renew. Energy 2016, 85, 1246-1259. [CrossRef]

20. Shan, X.B.; Deng, J.; Song, R.J.; Xie, T. A Piezoelectric Energy Harvester with Bending-Torsion Vibration in Low-Speed Water. Appl. Sci. Basel 2017, 7, 116. [CrossRef]

21. Okajima, A.; Yasui, S.; Kiwata, T.; Kimura, S. Flow-induced streamwise oscillation of two circular cylinders in tandem arrangement. Int. J. Heat Fluid Flow 2007, 28, 552-560. [CrossRef]

22. Zhou, Y.; So, R.M.C.; Liu, M.H.; Zhang, H.J. Complex turbulent wakes generated by two and three side-by-side cylinders. Int. J. Heat Fluid Flow 2000, 21, 125-133. [CrossRef]

23. Harichandan, A.B.; Roy, A. Numerical investigation of low Reynolds number flow past two and three circular cylinders using unstructured grid CFR scheme. Int. J. Heat Fluid Flow 2010, 31, 154-171. [CrossRef]

24. Chen, W.; Ji, C.; Williams, J.; Xu, D.; Yang, L.; Cui, Y. Vortex-induced vibrations of three tandem cylinders in laminar cross-flow: Vibration response and galloping mechanism. J. Fluids Struct. 2018, 78, $215-238$. [CrossRef]

25. Ding, L.; Zou, Q.F.; Zhang, L.; Wang, H. Research on Flow-Induced Vibration and Energy Harvesting of Three Circular Cylinders with Roughness Strips in Tandem. Energies 2018, 11, 2977. [CrossRef]

26. Zhou, Y.; Alam, M.M. Wake of two interacting circular cylinders: A review. Int. J. Heat Fluid Flow 2016, 62, 510-537. [CrossRef]

27. Mysa, R.C.; Kaboudian, A.; Jaiman, R.K. On the origin of wake-induced vibration in two tandem circular cylinders at low Reynolds number. J. Fluids Struct. 2016, 61, 76-98. [CrossRef]

28. Tamimi, V.; Naeeni, S.T.O.; Zeinoddini, M. Flow induced vibrations of a sharp edge square cylinder in the wake of a circular cylinder. Appl. Ocean Res. 2017, 66, 117-130. [CrossRef]

29. Pearcey, T.; Zhao, M.; Xiang, Y.; Liu, M. Vibration of two elastically mounted cylinders of different diameters in oscillatory flow. Appl. Ocean Res. 2017, 69, 173-190. [CrossRef]

30. Griffith, M.D.; Jacono, D.L.; Sheridan, J.; Leontini, J.S. Flow-induced vibration of two cylinders in tandem and staggered arrangements. J. Fluid Mech. 2017, 833, 98-130. [CrossRef]

31. Borazjani, I.; Sotiropoulos, F. Vortex-induced vibrations of two cylinders in tandem arrangement in the proximity-wake interference region. J. Fluid Mech. 2009, 621, 321-364. [CrossRef] [PubMed]

32. Liu, B.; Jaiman, R.K. Interaction dynamics of gap flow with vortex-induced vibration in side-by-side cylinder arrangement. Phys. Fluids 2016, 28, 127103. [CrossRef]

33. Liu, B.; Jaiman, R.K. Dynamics and stability of gap-flow interference in a vibrating side-by-side arrangement of two circular cylinders. J. Fluid Mech. 2018, 855, 804-838. [CrossRef]

34. Spalart, P.R.; Allmaras, S.R. A One-Equation Turbulence Model for Aerodynamic Flows. Recherché Aerospatiale 1994, 1, 5-21.

35. Zdravkovich, M.M. Flow around Circular Cylinders: Volume 1: Fundamentals; Oxford University Press: Oxford, UK, 2003.

(C) 2019 by the authors. Licensee MDPI, Basel, Switzerland. This article is an open access article distributed under the terms and conditions of the Creative Commons Attribution (CC BY) license (http://creativecommons.org/licenses/by/4.0/). 